\title{
Glycemic index, glycemic load, and pancreatic cancer risk (Canada)
}

\author{
Stephanie A.N. Silvera ${ }^{1, *}$, Thomas E. Rohan ${ }^{1}$, Meera Jain ${ }^{2}$, Paul D. Terry ${ }^{3}$, Geoffrey R. Howe ${ }^{4}$, Anthony \\ B. Miller ${ }^{2}$ \\ ${ }^{1}$ Department of Epidemiology and Population Health, Albert Einstein College of Medicine, 1300 Morris Park Avenue, \\ Bronx, NY 10461, USA; ${ }^{2}$ Department of Public Health Sciences, University of Toronto, Toronto, Canada; \\ ${ }^{3}$ Department of Epidemiology, Emory School of Public Health; ${ }^{4}$ Department of Epidemiology, Mailman School of \\ Public Health, Columbia University, New York, NY
}

Received 27 August 2004; accepted in revised form 18 October 2004

Key words: glycemic index, glycemic load, pancreatic neoplasms, prospective cohart.

\begin{abstract}
There is some evidence that plasma insulin and postload plasma glucose may be associated with risk of pancreatic cancer. Glycemic index and glycemic load are measures, which allow the carbohydrate content of individual foods to be classified according to their postprandial glycemic effects and hence their effects on circulating insulin levels. Therefore, we examined pancreatic cancer risk in association with glycemic index (GI), glycemic load (GL), and intake of dietary carbohydrate and sugar in a prospective cohort of 49,613 Canadian women enrolled in the National Breast Screening Study (NBSS) who completed a self-administered food frequency questionnaire between 1980 and 1985. Linkages to national cancer and mortality databases yielded data on cancer incidence and deaths, with follow-up ending between 1998 and 2000. During a mean 16.5 years of follow-up, we observed 112 incident pancreatic cancer cases. There was no association between overall glycemic index, glycemic load, total carbohydrate and total sugar intake and pancreatic cancer risk. In multivariate adjusted models, the hazard ratio (HR) for the highest versus lowest quartile levels of overall GI and GL were $1.43(95 \%$ confidence interval $[\mathrm{CI}]=0.56-3.65$, $\left.P_{\text {trend }}=0.58\right)$ and $0.80\left(95 \% \mathrm{CI}=0.45-1.41, P_{\text {trend }}=0.41\right)$, respectively. Our data suggest that overall glycemic index and glycemic load, as well as total sugar and total carbohydrate intake, are not associated with pancreatic cancer risk. However, given the limited literature regarding the role of diet in the etiology of pancreatic cancer, particularly with respect to glycemic index/load, further investigation is warranted.
\end{abstract}

\section{Introduction}

Relatively little is known about the etiology of pancreatic cancer. However, there is some evidence that plasma insulin levels might be relevant [1]. In an in vitro study, Fisher et al. [2] demonstrated that high-affinity insulin receptors are present in six pancreatic cell lines and also reported a dose-dependent proliferative response in these cell lines with increasing insulin concentrations. This observation is in accord with evidence that insulin

* Address Correspondence to: S.A.N. Silvera, Department of Epidemiology and Population Health, Albert Einstein College of Medicine, 1300 Morris Park Avenue, Room 1301, Bronx, NY10461, USA. Ph.: + 1718-430-3038; Fax:+1-178-430-8653; E-mail address: ssilvera@aecom. yu.edu is associated with the activation of mitogenic signals that stimulate pancreatic cell proliferation [3]. Furthermore, insulin receptor substrate-1 expression has been found to be increased in human pancreatic cancer [3]. Additional support for a role for insulin comes from the observation that postload plasma glucose levels were positively associated with pancreatic cancer risk in a cohort of men and women who were non-diabetic at baseline [4].

Glycemic index and glycemic load are measures which can be used to classify the carbohydrate content of individual foods according to their postprandial glycemic effects and hence their effects on blood insulin levels [5-8]. Using glycemic index values, the total glycemic effect of the diet (glycemic load) can be estimated [9]. 
Consumption of high GI diets has been associated with hyperinsulinemia $[5,9,10]$, while low GI diets have been shown to be associated with a lower postprandial rise in insulin [11], thus maintaining insulin sensitivity [12]. Therefore, glycemic index/load might be associated with pancreatic cancer risk. To date, however, it appears that the relationship between overall glycemic index and glycemic load and pancreatic cancer risk has been examined in only one epidemiological study [13]. In that investigation, a cohort study in women, there was no association between overall glycemic index and pancreatic cancer risk, although there was a statistically nonsignificant trend of increasing risk with increasing glycemic load intake [13]. Given the current lack of data regarding these relationships, we examined the association between overall glycemic index and glycemic load, as well as total carbohydrate and sugar intake (included because of their strong association with postprandial insulin response [14]), and pancreatic cancer risk in a cohort of Canadian women.

\section{Materials and methods}

The design of our study has been described in detail elsewhere [15]. Briefly, 89,835 women aged 40-59 years were recruited into the Canadian National Breast Screening Study (NBSS) between 1980 and 1985 from the general Canadian population [16]. At recruitment into the cohort, participants completed a self-administered questionnaire which sought information on demographic characteristics, lifestyle factors (including cigarette smoking), menstrual and reproductive history, and use of oral contraceptives and replacement estrogens. Starting in 1982, a self-administered food frequency questionnaire (FFQ) [17] was distributed to all new attendees at all screening centers, and to women returning to the screening centers for re-screening. The FFQ sought information on usual portion size and frequency of consumption of 86 food items, and included photographs of various portion sizes to assist respondents with quantifying intake. A comparison between the selfadministered questionnaire and a full interviewer-administered questionnaire, which has been subjected to both validity and reliability testing [17] and used in a number of epidemiologic studies [18], revealed that the two methods gave estimates of intake of the major macronutrients and dietary fiber which were moderately to strongly correlated with each other (reported correlation coefficients ranged from 0.47 to 0.72 ) [17]. A total of 49,613 dietary questionnaires were returned and available for analysis.
Glycemic index values of foods were obtained from published reports based on studies in North America [9]. Overall glycemic index was calculated by multiplying the carbohydrate content of a given food item by the number of servings per day of that food item and its glycemic index value, summing over all food items reported, and dividing by total carbohydrate intake. Total dietary glycemic load was calculated by multiplying the carbohydrate content of a given food item by the number of servings consumed per day and its glycemic index value and summing the values for all food items reported. When the reported glycemic index values for foods were observed to vary across studies [9] we used the mean of the reported values of glycemic index for that food.

Data from the completed self-administered food frequency questionnaires were used to estimate daily intake of nutrients using a database for Canadian foods that has been described elsewhere [18]. Briefly, nutrient values, including total carbohydrate, total fiber, and sugar intakes were calculated by using a data bank based on Handbook No. 8 of the US Department of Agriculture [19], modified and expanded for Canadian foods. The variable for total sugar was calculated by summing the nutrient intake values for galactose, glucose, fructose, lactose, sucrose, and maltose.

Data from the food frequency questionnaire were also used to estimate overall glycemic index and glycemic load. Glycemic index values of foods were obtained from published reports based on studies in North America [9]. Overall glycemic index was calculated by multiplying the carbohydrate content (in grams) of a given food item by the number of servings per day of that food item and its glycemic index value, summing over all food items reported, and dividing by the total carbohydrate in the diet. Total dietary glycemic load was calculated by multiplying the carbohydrate content of a given food item by the number of servings consumed per day and its glycemic index value and summing the values for all food items reported. Each unit increase in glycemic load represents the insulin response to the equivalent of 1 gram of glucose or carbohydrate from white bread (depending on the standard used) [20]. When the reported glycemic index values for foods were observed to vary across studies [9], we used the mean of the reported values of glycemic index for that food. The main foods contributing to glycemic load in the cohort are listed in a footnote to Table 1 .

Incident cases of pancreatic cancer and deaths from all causes were ascertained respectively by means of computerized record linkages to the Canadian Cancer Database and to the National Mortality Database, both 
Table 1. Baseline distributions of pancreatic cancer risk factors by quintiles of energy-adjusted glycemic load (GL)

\begin{tabular}{lllll}
\hline & \multicolumn{4}{l}{ Quartiles of energy-adjusted Glycemic Load (g/day) } \\
\cline { 2 - 5 } & $<125(n=12281)$ & $125-147(n=12281)$ & $148-169(n=12280)$ & $>169(n=12281)$ \\
\hline Mean overall glycemic index & $73.4(24.9)$ & $79.0(23.0)$ & $81.0(22.9)$ & $84.2(25.7)$ \\
Mean glycemic load (g/day) & $103.4(18.2)$ & $136.5(6.4)$ & $157.8(6.3)$ & $191.2(20.8)$ \\
Total carbohydrate (g/day) & $154.7(53.2)$ & $186.6(53.0)$ & $209.6(57.9)$ & $247.5(81.0)$ \\
Total sugar (g/day) & $61.8(19.6)$ & $77.6(19.3)$ & $85.6(20.5)$ & $99.7(29.6)$ \\
Total fiber (g/day) & $17.7(6.1)$ & $20.5(5.9)$ & $21.7(6.2)$ & $23.0(7.3)$ \\
Mean energy intake (kcal/day) & $2097(747)$ & $2029(589)$ & $2054(579)$ & $2106(647)$ \\
Mean age (years) & $48.1(5.5)$ & $48.5(5.6)$ & $48.7(5.6)$ & $48.9(5.7)$ \\
Mean BMI (kg/m $\left.{ }^{2}\right)$ & $25.1(4.4)$ & $25.0(5.1)$ & $24.6(4.3)$ & $24.4(4.6)$ \\
Pack-years of smoking (Mean) & $20.7(17.7)$ & $17.5(15.9)$ & $16.2(15.7)$ & $16.1(15.2)$ \\
Mean alcohol intake (g/day) & $15.2(19.7)$ & $8.9(11.1)$ & $6.3(8.8)$ & $3.8(6.3)$ \\
Some vigorous physical activity (\%) & 24.0 & 25.8 & 26.0 & 24.2 \\
HRT use (\% ever) & 25.2 & 24.7 & 25.0 & 25.2 \\
Live births & $2.6(2.8)$ & $2.6(2.6)$ & $2.6(3.0)$ & $2.6(2.6)$ \\
\hline
\end{tabular}

${ }^{\text {a }}$ The main foods contributing to glycemic load in the cohort include white bread (sliced), rolls, muffins, potatoes (baked, boiled, and mashed), French fries, cakes, cookies, rice, pasta, pizza, cold breakfast cereals, pies and tarts, cola, other soft drinks, citrus fruits and juices and other fruits, crisp snacks (such as potato chips or popcorn), candy, chocolate, peas, beans and lentils, hot breakfast cereals, dark and wholegrain breads, corn, root vegetables other than potatoes, jam, jelly and honey, sugar in tea or coffee, ice cream, and peanut butter.

${ }^{\mathrm{b}}$ Numbers in parentheses represent the standard deviation.

of which are maintained by Statistics Canada. The linkages to the databases yielded data on cancer incidence and mortality to 31 December 2000 for women in Ontario, 31 December 1998 for women in Quebec, and 31 December 1999 for women in other provinces. There is evidence that the use of record linkages to ascertain incident cancer cases and deaths in Canada is both complete and accurate [21, 22].

Of the 46,613 women who had returned a FFQ and had provided information on energy intake, we excluded 88 women with extreme energy intake values (at least three standard deviations above or below the mean value for $\log _{\mathrm{e}}$ caloric intake). Additionally, we excluded one woman with prevalent pancreatic cancer at baseline. Analyses were thus based on 49,111 women for whom complete information on all covariates was available.

Cox proportional hazards models (using age as the time scale) were used to estimate hazard ratios (HR) and $95 \%$ confidence intervals $(\mathrm{CI})$ for the association between energy-adjusted quartile levels of glycemic load, overall glycemic index, total carbohydrates, sucrose, fructose, and total sugar, and pancreatic cancer risk; energy adjustment was performed using the residual method [23]. For these analyses, cases contributed person-time to the study from their date of enrollment until the date of diagnosis of their pancreatic cancer, and non-cases contributed person-time from their date of enrollment until the termination of follow-up (the date to which cancer incidence data were available for women in the corresponding province) or death, whichever occurred earlier. Multivariate models included body mass index [defined as weight $(\mathrm{kg}) /$ height $\left(\mathrm{m}^{2}\right)$; weight and height were measured at baseline [24]], self-reported alcohol consumption (frequency of consumption of beer, wine, and liquor) and smoking history (in pack years, defined as the number of cigarettes per day multiplied by how many years they reported smoking), energy intake (kcal/day), study center and randomization group. To test for trend we fitted the median value of each quartile as an ordinal variable in the risk models, and evaluated the statistical significance of the coefficient using the Wald test [25]. All analyses were performed using SAS version 8 (SAS Institute Cary, NC).

\section{Results}

The average duration of follow-up for cohort members was 16.5 (809,492 person-years), during which 112 cases of pancreatic cancer were diagnosed. The mean (S.D.) age at diagnosis for the cases was $61.7( \pm 7.3)$ years. For the cohort as a whole, the means ( \pm SD) of the energyadjusted overall glycemic index and glycemic load were $79.4( \pm 24.5)$ and $147.2( \pm 35.1) \mathrm{g} /$ day, respectively.

There was a two-fold variation in mean glycemic load values between the lowest and highest quintile levels (Table 1). Compared to those with relatively low glycemic load values, women with high glycemic load values reported lower alcohol consumption, fewer pack-years 
of smoking, and consumed more total calories, total carbohydrates, sugar and fiber (Table 1). No appreciable variation was observed in use of hormone replacement therapy (HRT), participation in vigorous physical activity, mean body mass index (BMI), or parity by quartile levels of glycemic load. The patterns for overall glycemic index were similar to those for the glycemic load (data not shown).

Table 2 shows that in age- and energy-adjusted models, there was no association between glycemic load, overall glycemic index, fructose consumption, or total sugar intake and risk of pancreatic cancer. Although there was an inverse association of statistical significance between age- and energy-adjusted total carbohydrate intake and pancreatic cancer risk $(\mathrm{HR}=0.49,95 \% \mathrm{CI}=0.25-0.94$, $\left.P_{\text {trend }}=0.05\right)$ and between sucrose consumption and pancreatic cancer risk $(\mathrm{HR}=0.55,95 \% \mathrm{CI}=0.31-0.97$, $\left.P_{\text {trend }}=0.02\right)$, additional adjustment for other potential confounders attenuated the associations and rendered them statistically non-significant. Additional adjustment for total fiber consumption had essentially no impact on the hazard ratios (data not shown).

Table 2. Adjusted ${ }^{\text {a }}$ hazard ratios and $95 \%$ confidence intervals (CI) for the association between quartiles of overall glycemic index, glycemic load, total carbohydrate, total sugar, and total fiber and risk of pancreatic cancer

\begin{tabular}{|c|c|c|c|}
\hline & \multirow[t]{2}{*}{ Cases/person-years } & \multicolumn{2}{|l|}{ Hazard ratio $(95 \% \mathrm{CI})$} \\
\hline & & Age- \& energy adjusted & Multivariate adjusted \\
\hline \multicolumn{4}{|c|}{ Overall glycemic index } \\
\hline$<63$ & $35 / 203,226$ & 1.0 (referent) & 1.0 (referent) \\
\hline $63-73$ & $28 / 202,451$ & $1.01(0.54-1.86)$ & $1.29(0.67-2.47)$ \\
\hline 74-92 & $26 / 202,337$ & $1.03(0.50-2.13)$ & $1.31(0.60-2.83)$ \\
\hline$>92$ & $23 / 201,479$ & $1.07(0.44-2.59)$ & $1.43(0.56-3.65)$ \\
\hline$P$ for trend & & 0.86 & 0.58 \\
\hline \multicolumn{4}{|c|}{ Glycemic Load (g/day) } \\
\hline$<125$ & $33 / 201,976$ & 1.0 (referent) & 1.0 (referent) \\
\hline $125-147$ & $29 / 202,564$ & $0.88(0.53-1.45)$ & $0.95(0.56-1.61)$ \\
\hline $148-169$ & $22 / 202,274$ & $0.73(0.43-1.23)$ & $0.88(0.51-1.51)$ \\
\hline$>169$ & $25 / 202,678$ & $0.71(0.42-1.20)$ & $0.80(0.45-1.41)$ \\
\hline$P$ for trend & & 0.15 & 0.41 \\
\hline \multicolumn{4}{|c|}{ Total Carbohydrate (g/day) } \\
\hline$<152$ & $35 / 203,208$ & 1.0 (referent) & 1.0 (referent) \\
\hline $152-191$ & $18 / 202,520$ & $0.42(0.23-0.75)$ & $0.49(0.28-0.90)$ \\
\hline $192-236$ & $25 / 202,504$ & $0.49(0.28-0.86)$ & $0.60(0.33-1.07)$ \\
\hline$>236$ & $34 / 201,259$ & $0.49(0.25-0.94)$ & $0.63(0.31-1.26)$ \\
\hline$P$ for Trend & & 0.05 & 0.23 \\
\hline \multicolumn{4}{|c|}{ Total Sugar (g/day) } \\
\hline$<64$ & $32 / 202,211$ & 1.0 (referent) & 1.0 (referent) \\
\hline $64-79$ & $26 / 202,450$ & $0.78(0.46-1.31)$ & $0.72(0.42-1.257)$ \\
\hline $79-96$ & $22 / 202,684$ & $0.65(0.38-1.12)$ & $0.73(0.42-1.28)$ \\
\hline$>96$ & $32 / 202,147$ & $0.93(0.57-1.52)$ & $0.99(0.59-1.66)$ \\
\hline$P$ for Trend & & 0.65 & 1.00 \\
\hline \multicolumn{4}{|l|}{ Sucrose (g/day) } \\
\hline$<17$ & $34 / 202,814$ & 1.0 (referent) & 1.0 (referent) \\
\hline $17-24$ & $28 / 202,752$ & $0.71(0.43-1.18)$ & $0.78(0.46-1.32)$ \\
\hline $25-34$ & $20 / 202,349$ & $0.45(0.25-0.79)$ & $0.55(0.30-0.98)$ \\
\hline$>34$ & $30 / 201,576$ & $0.55(0.31-0.97)$ & $0.64(0.35-1.17)$ \\
\hline$P$ for Trend & & 0.02 & 0.09 \\
\hline \multicolumn{4}{|l|}{ Fructose (g/day) } \\
\hline$<13$ & $23 / 202,002$ & 1.0 (referent) & 1.0 (referent) \\
\hline $13-19$ & $27 / 202,652$ & $1.06(0.60-1.85)$ & $1.20(0.68-2.11)$ \\
\hline $20-25$ & $28 / 202,860$ & $1.02(0.58-1.79)$ & $1.22(0.68-2.16)$ \\
\hline$>25$ & $34 / 201,978$ & $1.17(0.66-2.05)$ & $1.18(0.65-2.13)$ \\
\hline$P$ for Trend & & 0.62 & 0.62 \\
\hline
\end{tabular}

${ }^{\mathrm{a}}$ Multivariable models included age (as time to event variable), BMI in $\mathrm{kg} / \mathrm{m}^{2}(<25,25-29$, $>30)$, alcohol (zero plus four levels of intake), smoking (pack-years, quartiles), parity (nulliparity plus 3 levels for parous), energy intake (continuous), study center, and randomization group. 


\section{Discussion}

High glycemic index diets are associated with increased insulin secretion $[5,9,10,26]$, which has been shown to promote pancreatic cancer cell growth in vitro [27], and has been hypothesized to affect pancreatic cancer risk by several mechanisms, including alteration of cell cycle kinetics (insulin facilitates the transit of cells through the G1 phase of the cell cycle) [28], inhibition of apoptosis [29], and down-regulation of insulin-like growth factor binding protein 1 (IGFBP-1) [30, 31].

In the prospective study reported here, there was no association between either overall glycemic index or glycemic load and pancreatic cancer risk during a 16-year follow-up period. Although a statistically significant inverse association was found with total carbohydrate consumption in the age- and energyadjusted model, this association was attenuated in the multivariate model. By using a prospective study design, recall bias was avoided, but misclassification of dietary intake may have attenuated the observed associations. A limitation of our study is the use of a one-time dietary assessment that may not have been representative of the dietary habits of the study participants over the course of follow-up. In addition, the small number of pancreatic cancer cases precluded any subgroup analyses. As well, the lack of statistical significance in our multivariate models may be due to a lack of power due to the small number of cases.

Only two prospective studies have previously examined the association between glycemic index/load, dietary sugar, or dietary fiber and pancreatic cancer risk. Stolzenberg-Solomon et al. [32], analyzed data from the Alpha-Tocopherol, Beta-Carotene Cancer Prevention Study cohort in Finland, in which 163 incident pancreatic cancer cases were observed during 13 years of follow-up, and reported a statistically significant inverse trend of pancreatic cancer risk with carbohydrate consumption among male smokers. This study population is clearly different from our cohort of Canadian women and therefore these results are not directly comparable to ours. In contrast, Michaud et al. [13], analyzed data from the Nurses' Health Study $(n=88,802)$, in which 180 incident pancreatic cancer cases were found during 18 years of follow-up . Our overall results are similar to those of Michaud et al. [13], in that there was no association overall with either glycemic index or glycemic load, but we were unable to confirm their finding of a statistically significant trend of increasing risk with increasing glycemic load among women with a body mass index of at least $25 \mathrm{~kg} / \mathrm{m}^{2}$, because the relatively small number of cases in our study precluded stratified analyses. Furthermore, unlike
Michaud et al.[13], we did not collect data on diabetes, and therefore were unable to adjust for it in the analysis. However, although there is evidence from epidemiologic studies to support a causal association between diabetes mellitus and pancreatic cancer [33], it is not immediately obvious that failure to adjust for diabetes would have confounded the associations observed here. Specifically, in the 1980's dietary recommendations for diabetics stressed high intake of complex carbohydrates and fiber and low fat consumption [34, 35]. These recommendations were similar to those commonly promoted at that time for the population as a whole [36]. Hence, it is unlikely that there were any substantial differences in dietary patterns for diabetics and non-diabetics during the period in which dietary data were collected for the present study. Nevertheless, if those diagnosed with diabetes had altered their diet to (for example) include more foods with low glycemic index values, then it is possible that our inability to adjust for diabetes could have obscured a positive association between glycemic index/load and pancreatic cancer risk.

Evidence regarding the role of diet in the etiology of pancreatic cancer is limited [37], particularly with respect to the role of glycemic index/load. However, given that diet is a potentially modifiable exposure, the possibility that high glycemic load diets might be associated with increased pancreatic cancer risk warrants further investigation.

\section{Acknowledgements}

We thank Statistics Canada, the provincial and territorial Registrars of Vital Statistics, and the Cancer Registry directors for their assistance in making the cancer incidence and mortality data available.

\section{References}

1. Ekbom A, Hunter D (2002) Pancreatic cancer. In: Adami H, Hunter D, and Trichopoulos D, eds. Textbook of Cancer Epidemiology. New York, NY: Oxford University Press.

2. Fisher WE, Laszlo GB, Schirmer WJ (1996) Insulin promotes pancreatic cancer: evidence for endocrine influence on exocrine pancreatic tumors. J Surg Res 63: 310-313.

3. Gupta K, Krishnaswamy G, Karnad A, Peiris A (2002) Insulin: a novel factor in carcinogenesis. Am J Med Sci 232: 140-145.

4. Gapstur SM, Gann PH, Lowe W, et al. (2000) Abnormal glucose metabolism and pancreatic cancer mortality. J Am Med Assoc 283: 2552-2558.

5. Holt SH, Miller JC, Petocz P (1997) An insulin index of foods: the insulin demand generated by $1000-\mathrm{kJ}$ portions of common foods. Am J Clin Nutr 66: 1264-1276. 
6. Ionescu-Tirgoviste C, Popa E, Sintu E, et al. (1983) Blood glucose and plasma insulin responses to various carbohydrates in type 2 (non-insulin-dependent diabetes). Diabetologia 24: 80-84.

7. Jenkins DJ, Kendall CW, Augustin LS, et al. (2002) Glycemic index: overview of implications in health and disease. Am J Clin Nutr 76: 266S-273S.

8. Ludwig DS (2002) The glycemic index: physiological mechanisms relating to obesity, diabetes, and cardiovascular disease. $\mathrm{J} \mathrm{Am} \mathrm{Med}$ Assoc 287: 2414-2423.

9. Foster-Powell K, Holt SH, Brand-Miller JC (2002) International table of glycemic index and glycemic load values: 2002. Am J Clin Nutr 76: 5-56.

10. Byrnes SE, Miller JCB, Denyer GS (1995) Amylopectin starch promotes the development of insulin resistance in rats. $J$ Nutr $\mathbf{1 2 5}$ $1430-1437$.

11. Ludwig DS (2000) Dietary glycemic index and obesity. $J$ Nutr 130: 280S-283S.

12. Plymate SR, Jones RE, Matej LA, Friedl KA (1988) Regulation of sex hormone binding globulin (SHBG) production in Hep G2 cells by insulin. Steroids 52: 339-340.

13. Michaud DS, Liu S, Giovannucci E, et al. (2002) Dietary sugar, glycemic load, and pancreatic cancer risk in a prospective study. J Natl Cancer Inst 94: 1293-1300.

14. Jenkins D, Wolever TM, Buckley G, et al. (1988) Low-glycemicindex starchy foods in the diabetic diet. Am J Clin Nutr 48 248-254.

15. Terry P, Jain M, Miller AB, Howe GR, Rohan TE (2002) Dietary carotenoid intake and colorectal cancer risk. Nutr Cancer $\mathbf{4 2}$ $167-172$.

16. Miller AB, Baines CJ, To T, Wall C (1992) Canadian National Breast Screening Study. I. Breast cancer detection and death rates among women aged 40-49 years. II. Breast cancer detection and death rates among women aged 50-59 years. Can Med Assoc J 147: 1459-1488.

17. Jain MG, Harrison L, Howe GR, Miller AB (1982) Evaluation of a self-administered dietary questionnaire for use in a cohort study. Am J Clin Nutr 36: 931-935.

18. Morgan AB, Jain M, Miller AB, et al. (1978) A comparison of dietary methods in epidemiologic studies. Am J Epidemiol 107: 488-498.

19. Watt BK, Merrill AL. (1963, expansion March 1972) Composition of foods: raw, processed, prepared. USDA Handbook No. 8 . Washington, DC: Agriculture Research Service.

20. Liu S, Manson JE, Stampfer MJ, et al. (2001) Dietary glycemic load assessed by food-frequency questionnaire in relation to plasma high-density-lipoprotein cholesterol and fasting plasma triacylglycerols in postmenopausal women. Am J Clin Nutr 73 : $560-566$.

21. Robles SC, Marrett LD, Clarke EA, Risch HA (1988) An application of capture-recapture methods to the estimation of completeness of cancer registration. J Clin Epidemiol 41: 495-501.
22. Shannon HS, Jamieson E, Walsh C, et al. (1989) Comparison of individual follow-up and computerized record linkage using the Canadian Mortality Data Base. Can J Public Health 80: $54-57$.

23. Willett WC, Stampfer MJ (1986) Total energy intake: implications for epidemiologic analyses. Am J Epidemiol 124: 17-27.

24. Jain M, Miller AB (1997) Tumor characteristics and survival of breast cancer patients in relation to premorbid diet and body size. Breast Cancer Res Treat 42: 43-55.

25. Rothman K, Greenland S (1998) Modern Epidemiology 2nd edn. Philadelphia (PA): Lippincott-Raven; p. 319.

26. Kaaks R, Lukanova A (2002) Effects of weight control and physical activity in cancer prevention: role of endogenous hormone metabolism. Ann N Y Acad Sci 963: 268-281.

27. Fisher WE, Boros LG, Schirmer WJ (1996) Insulin promotes pancreatic cancer: evidence for autocrine and paracrine roles. J Surg Res 63: 310-313.

28. Gross G, Boldt DH, Osborne CK (1984) Perturbation by insulin of human breast cancer cell kinetics. Cancer Res 44: 35703575 .

29. Geier A, Beery R, Haimshon M, Hemi R, Lunenfeld B (1992) Serum and insulin inhibit cell death induced by cycloheximide in the human breast cancer cell line MCF-7. In Vitro Cell Dev Biol 28A: $415-418$.

30. Musey VC, Goldstein S, Farmer PK, Moore PB, Phillips LS (1993) Differential regulation of IGF-1 and IGF-binding protein-1 by dietary composition in humans. Am J Med Sci 305: 131138 .

31. Suikkari AM, Koivisto VA, Rutanen EM, et al. (1988) Insulin regulates the serum levels of low molecular weight insulin-like growth factor-finding protein. $J$ Clin Endocrinol Metab 66: 266-272.

32. Stolzenberg-Solomon RZ, Pietinen P, Taylor PR, Virtamo J, Albanes D (2002) Prospective study of diet and pancreatic cancer in male smokers. Am J Epidemiol 155: 783-192.

33. Michaud DS (2004) Epidemiology of pancreatic cancer. Minerva Chir 59: 99-111.

34. American Diabetes Association (1979) Principles of nutrition and dietary recommendations for individuals with diabetes mellitus. Diabetes 28: 1027.

35. (1982) Dietary recommendations for diabetics for the 1980s-a policy statement by the British Diabetic Association: prepared by the Nutrition Sub-Committee of the British Diabetic Association's Medical Advisory Committee. Hum Nutr Appl Nutr 36: 378, 380, 382

36. (1986) Statement on nutrition and your health: dietary guidelines for Americans. J Am Diet Assoc 86: 107-108.

37. World Cancer Research Fund (1997) Food, Nutrition and the Prevention of Cancer: A Global Perspective. Washington DC: American Institute for Cancer Research. 